\title{
Aspectos epidemiológicos da Leishmaniose Visceral Canina e Humana no estado do Maranhão, Brasil (2009-2012)*
}

\section{Epidemiological aspects of Human and Canine Visceral Leishmaniasis in the state of Maranhão, Brazil (2009-2012)}

\author{
Daniela Pinto Sales, ${ }^{* *}$ Daniel Praseres Chaves, ${ }^{* *}$ Nathálya dos Santos Martins, ${ }^{* *}$ Maria Inez Santos Silva, ${ }^{* *}$
}

\begin{abstract}
Resumo
Este trabalho objetivou realizar um estudo retrospectivo sobre os aspectos epidemiológicos da Leishmaniose Visceral Canina (LVC) e Humana (LVH) no estado do Maranhão no período de 2009-2012. A amostra constituiu-se dos dados de fichas de registros provenientes de animais do Centro de Controle de Zoonose (CCZ) de São Luís-MA e dos registros notificados na Secretaria de Estado da Saúde (SES) no período de 2009 a 2012. Foram registrados um total de 2.259 atendimentos a caninos com suspeita de Leishmaniose no CCZ, a maior frequência de registros foi de machos 1.198 (53,03\%). Desses atendimentos, $564(24,96 \%)$ foram soropositivos para Leishmania e a maior frequência ocorreu no período seco $326(57,80 \%)$. Dos casos de LVH, foram notificados 2.755 , destes $1.577(57,24 \%)$ foram confirmados e $59(3,74 \%)$ vieram ao óbito. Dos casos notificados para a LVH no estado do Maranhão, a maioria era do sexo masculino 1.656 (60,12\%). A faixa etária onde houve maior ocorrência de casos notificados estava compreendida entre 1-4 anos de idade com 282 (37,95\%) em 2011. Verificou-se que a LVH estava presente em 129 municípios do estado do Maranhão, sendo que os de maior frequência foram Imperatriz com 126 (4,57\%). Dessa forma, pode-se concluir que ocorreu elevado número de casos de Leishmaniose Visceral Canina e Humana no estado do Maranhão no período pesquisado.
\end{abstract}

Palavras-chave: epidemiologia, Leishmania, frequência

\section{Abstract}

This study aimed to conduct a retrospective study on the epidemiology of Canine Visceral Leishmaniasis (CVL) and Human (LVH) in the state of Maranhão in the period 2009-2012. The sample consisted of data sheets records from animals from Zoonosis Control Center (CCZ) of São Luis - MA and records reported in the State Department of Health (SES) from 2009 to 2012. We recorded a total of 2.259 calls to canines with suspected leishmaniasis in the CCZ, the largest attendance records was 1.198 males $(53,03 \%)$. Of these calls, 564 (24,96\%) were seropositive for Leishmania and the highest prevalence occurred in the dry period $326(57,80 \%)$. Of the cases reported to the LVH in the state of Maranhão, most were male $1.656(60,12 \%)$. The age group where there was a higher incidence of reported cases was between 1-4 years old with $282(37,95 \%)$ in 2011. It was found that LVH was present in 129 municipalities in the state of Maranhão, with the highest prevalence were Imperatriz with $126(4,57 \%)$. Thus, it can be concluded that there was high number of cases of Canine Visceral Leishmaniosis and Human in the state of Maranhão in the period surveyed.

Keywords: epidemiology, Leishmania, frequency

\section{Introdução}

A Leishmaniose é causada por um protozoário do gênero Leishmania, cujo ciclo biológico heteroxênico tem a participação de um hospedeiro vertebrado (principalmente homem e canídeos), além de um invertebrado (fêmeas do gênero Lutzomyia longipalpis). Os principais reservatórios da leishmaniose visceral são os cães domésticos, porém podem ser encontrado naturalmente também em animais silvestres (raposado-mato, cachorro-do-mato, gambás), equídeos, roedores e o gato, (SCHIMMING e SILVA, 2012).

Os sintomas da leishmaniose canina são diversos, podendo observar-se linfoadenomegalia, onicogrifose, anemia, hepato e esplenomegalia, lesões cutâneas, trombose, epistaxe, lesões oculares e poliartrites, dentre outros (FARIA e ANDRADE, 2012). Os animais podem permanecer assintomáticos (representando cerca de 20 a $40 \%$ de uma população soropositiva) por toda a vida ou desenvolver sintomas após períodos que variam de três meses a alguns anos (IKEDA-GARCIA e MARCONDES, 2007).

O período de incubação da leishmaniose é bastante variável, no homem varia de 10 dias a 24 meses, com média entre 2 a 6 meses. No cão, varia de 3 meses a vários anos com média de 3 a 7 meses (BRASIL, 2006).

Aleishmaniose visceral apresenta uma importância significativa no contexto epidemiológico em decorrência do processo de urbanização e das alterações no ambiente natural. É uma

*Recebido em 19 de novembro de 2015 e aceito em 10 de dezembro de 2017.
${ }^{* *}$ Universidade Estadual do Maranhão/UEMA, São Luís, Maranhão, Brasil.
Autor para correspondência - Daniel Praseres Chaves. E-mail: daniel@cernitas.com.br 
doença grave e fatal tendo apresentado um crescimento muito significativo nos últimos anos, sendo um caso preocupante para a saúde pública (COSTA, 2011). Conforme Monteiro et al. (2005) os principais determinantes da leishmaniose visceral nos grandes centros são: convívio muito próximo do homem com o principal reservatório (cão), aumento da densidade do vetor, desmatamento acentuado e o constante processo migratório.

Conforme Nascimento-Rocha et al. (2002) a Leishmaniose é uma antropozoonose primeiramente descrita em 1903, na Índia, por Leishman e Donovan, apresenta uma incidência anual de aproximadamente 2 milhões de casos em todo o mundo. A doença ocorre em todo continente americano com $87 \%$ dos casos registrados no Brasil, apresentando uma significativa expansão geográfica desde 1999, com ocorrência de casos em 23 estados brasileiros, sendo a maior incidência encontrada nas regiões Nordeste e Norte (NASCIMENTO-ROCHA et al., 2002; CAMARGO e BONDAN, 2015).

Nas Américas os países mais acometidos pela leishmaniose visceral são: Brasil (90\% dos casos), Colômbia, Venezuela, Bolívia, EI Salvador e Honduras (VERONESI, FOCACCIA, 2003). Atualmente no Brasil apresenta ampla distribuição territorial, com aspectos geográficos, climáticos e sociais diferenciados, se expandindo para áreas urbanas mudando seu perfil epidemiológico, antes eminentemente rural.

Os estados da região Nordeste possuem a maior frequência de casos de LV, com destaque para Maranhão e Piauí (os mais pobres da região), Bahia, Ceará e Rio Grande do Norte. A seca nesta região agravou o quadro social, e com o êxodo rural, a presença de migrantes nas periferias urbanas contribuiu como fonte de infecção de indivíduos susceptíveis (COSTA et al., 1995).

No estudo realizado por Penha et al. (2013) na cidade de São Luís-MA, a espécie mais abundante foi $L$. longipalpis $(53,5 \%)$ e L. whitmani $(40,7 \%)$, a frequência dos flebotomíneos foi maior no peridomicílio $(94,5 \%)$, a doença vem sendo notificados na zona rural e em áreas periurbanas. Estima-se que, para cada caso humano, há uma média de pelo menos 200 cães infectados, sendo este o principal reservatório da doença para o ser humano, sendo os casos em humanos frequentemente são antecedidos de casos caninos (NEVES et al., 1997).

$\mathrm{Na}$ cidade de São Luís-MA, em 1982 houve um registro de 1.089 casos em quinze anos de LV devido ao êxodo rural e a migração de lavradores de Estados vizinhos. Em seguida, houve expansão para outras áreas da Ilha de São Luís, como São José de Ribamar e Paço do Lumiar (MENDES et al., 2002). No estudo de Barbosa et al. (2010) na cidade de São Luís - MA a análise sorológica para Leishmaniose Visceral demonstrou que, das 100 amostras analisadas de cães, 67\% apresentaram resultados positivos para anticorpos IgG anti-Leishmania.

Dessa forma, este trabalho objetivou realizar um estudo retrospectivo sobre os aspectos epidemiológicos da Leishmaniose Visceral Canina (LVC) na cidade de São Luis, e a Leishmaniose
Visceral Humana (LVH) no Estado do Maranhão no período de 2009-2012.

\section{Material e Métodos}

A pesquisa caracterizou-se por um estudo epidemiológico, retrospectivo, realizada no período de 2009 a 2012.

A amostra constituiu-se de dados de fichas de registros provenientes de animais do Centro de Controle de Zoonose (CCZ) de São Luís e dos registros notificados da doença na Secretaria de Estado da Saúde (SES) no período de 2009 a 2012, por não haver contato direto com animais e/ou seres humanos (somente fichas de registros) não houve a necessidade de licença de Comitê de ética.

Foi avaliado o número de casos positivos e negativos de Leishmaniose Visceral (LV), a procedência e época do ano da ocorrência desses casos, o método de diagnóstico e as seguintes variáveis epidemiológicas: sexo e idade. Outras variáveis importantes, tais como a profissão, que apresenta uma grande influência na transmissão do protozoário, a presença de cão doméstico ou errante e a idade dos cães, o que pode ser muito significante na epidemiologia da doença não estavam presentes nas fichas de registros.

Os dados coletados desta pesquisa foram tabulados em planilhas do programa Microsoft Office Excel 2010, utilizandose cálculos e construção de tabelas. A análise dos dados foi realizada através da ANOVA e teste de Tukey-Kramer na comparação das médias.

Conforme o banco de dados utilizado neste estudo, o diagnóstico para a LV utilizada nos cães e nos humanos foi a Reação de Imunofluorescência Indireta (RIFI), esta é a recomendadas pelo Ministério da Saúde para o inquérito epidemiológico canino e humano por ser de fácil execução, rapidez, baixo custo e sensibilidade e especificidade adequadas. Estes exames são realizados em laboratório central estadual (LACENs) e no Centro de Controle de Zoonoses (CCZs).

\section{Resultado e Discussão}

\section{Leishmaniose Visceral Canina (LVC)}

No período de estudo (2009-2012) foram registrados no CCZ um total de 2.259 atendimentos a caninos com suspeita de Leishmaniose, sendo 53,03\% (1.198) machos e 47,97\% (1.061) fêmeas.

Desses atendimentos, 564 (24,96\%) foram soropositivos para a Leishmania, sendo 307 (54,43\%) machos e 257 (45,57\%) fêmeas.

No ano de 2009 houve 23,5\% (134) animais soropositivos, em 2010 23,8\% (102), em 2011 27,4\% (180) e em 2012 24,6\% (148). A figura 1 apresenta o total de animais soropositivos conforme o sexo em cada ano do estudo. 
Os dados acima corroboram com o estudo de Matos et al. (2006), pois dos 39 cães positivos para a LVC na cidade de Mossoró - RN, 19 $(46,1 \%)$ eram fêmeas e $20(51,3 \%)$ machos. Ciaramella e Corona (2003), afirmam que não existe predisposição de sexo, sugerindo que esta maior predominância de machos pode está associada ao temperamento mais agressivo, maior domínio territorial e maior porte comparativamente as fêmeas, e serem mais frequentemente utilizados como animais de guarda, permanecendo por isso mais tempo no exterior dos domicílios.

Verificou-se que dos animais soropositivos para Leishmania o maior número de procedência destes foi do Distrito do Tirirical em São Luís - MA em todos os períodos de abrangência do estudo, seguido do Distrito da Cohab. Houve diferença estatística entre os soropositivos e os distritos sanitários Tirirical, Cohab e Vila Esperança $(P>0,05)$ (teste de Tukey) (Figura 2).

No estudo realizado por Amora et al. (2006), foram identificados 28 (45\%) dos cães soropositivos para anticorpos IgG anti- $L$. chagasi na área rural e 47 (34\%) cães na área urbana. Esse fato mostra que esta zoonose está se urbanizando. Conforme Monteiro et al. (2005) os inquéritos sorológicos na população de cães e os levantamentos entomológicos nas áreas endêmicas, revelam a prevalência da LVC muito alta e a presença predominante do vetor, o que redunda em elevado risco de transmissão para o homem. As localidades próximas de matas, com presença de animais silvestres e com ausência de saneamento básico, apresentam uma tendência para abundância de vetores e reservatórios, o que contribui para a uma alta soroprevalência canina (GUIMARÃES et al., 2005).

O Distrito Tirirical apresentou a maior prevalência de casos $(52,39 \%)$ de LVC. O estudo realizado por Barbosa et al. (2010) na busca de variáveis epidemiológicas da LVC no município de São Luís - MA, as localidades que apresentaram maior quantidade de casos positivos foram as pertencentes ao distrito do Tirirical: Cajupari, Cruzeiro de Santa Bárbara e Cidade Operária com 94\%, 81\% e $67 \%$, respectivamente.

Verificou-se que dos animais soropositivos para Leishmania a maior frequência ocorreu no período seco $326(57,80 \%)$, enquanto o período chuvoso prevaleceu 238 (42,20\%) (Tabela 3).

$\mathrm{O}$ inseto transmissor tem atividade máxima durante os meses de Primavera e Verão (CIARAMELLA e CORONA, 2003). Porém, deve-se observar o período de incubação da doença, que pode variar de meses a vários anos, não havendo por isso uma relação direta entre o período de infecção e o aparecimento de doença clínica.

\section{Soropositivos conforme sexo}

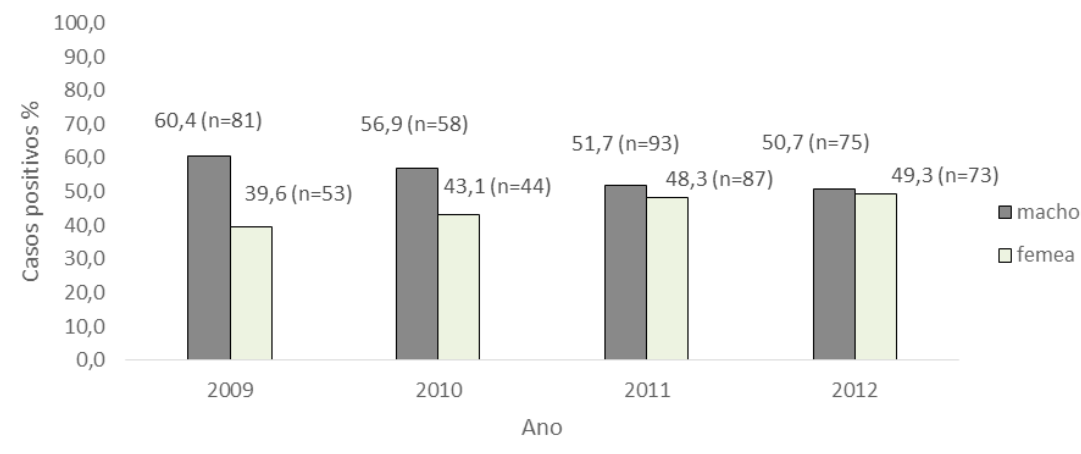

Figura 1: Distribuição dos cães soropositivos à Reação de Imunofluorescência Indireta (RIFI) para a Leishmania na cidade de São Luís - MA, segundo sexo, no período de 2009 a 2012.

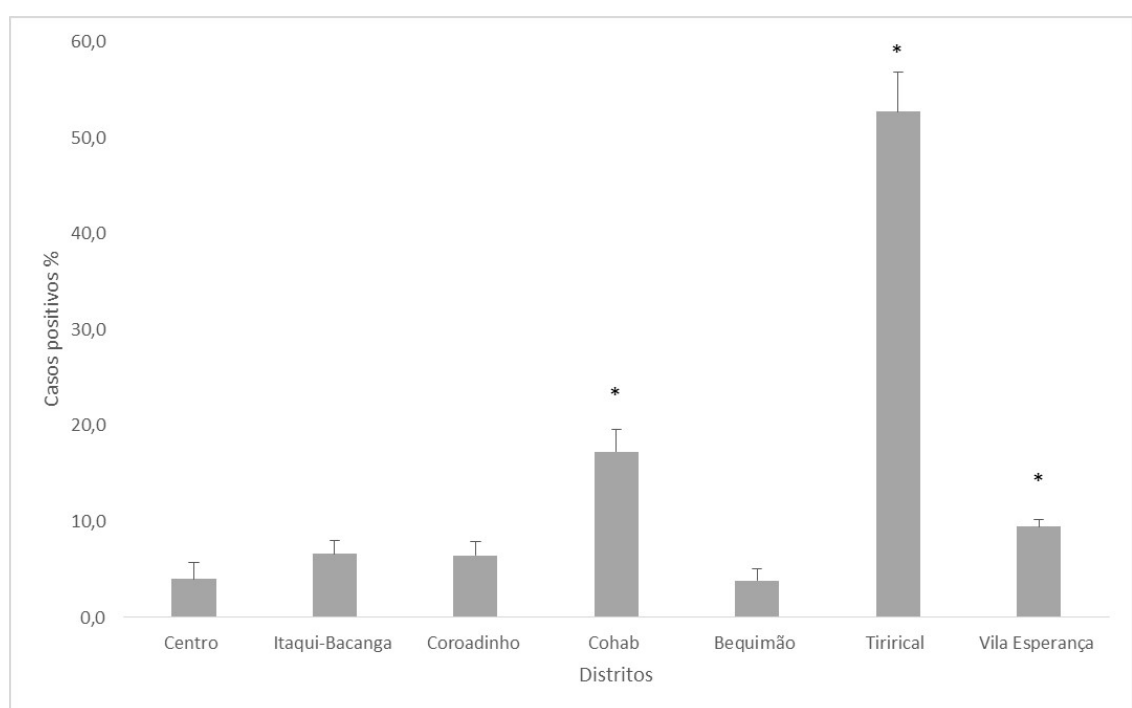

Figura 2: Distribuição dos cães soropositivos à Reação de Imunofluorescência Indireta (RIFI) para a Leishmania na cidade de São Luís - MA, segundo Distrito Sanitário, no período de 2009 a 2012.

Tabela 1: Distribuição dos cães soropositivos à Reação de Imunofluorescência Indireta (RIFI) para a Leishmania na cidade de São Luís - MA, segundo época do ano, no período de 2009 a 2012

\begin{tabular}{cccc}
\hline Anos & $\begin{array}{c}\text { Período chuvoso } \\
\text { N (\%) }\end{array}$ & $\begin{array}{c}\text { Período seco } \\
\text { N (\%) }\end{array}$ & $\begin{array}{c}\text { Total } \\
\text { N (\%) }\end{array}$ \\
\hline 2009 & $57(42,54 \%)$ & $77(57,46 \%)$ & $134(23,76 \%)$ \\
2010 & $37(36,27 \%)$ & $65(63,73 \%)$ & $102(18,09 \%)$ \\
2011 & $77(42,78 \%)$ & $103(57,22 \%)$ & $180(31,91 \%)$ \\
2012 & $67(45,27 \%)$ & $81(54,73 \%)$ & $148(26,24 \%)$ \\
Total & $238(42,20 \%)$ & $326(57,80 \%)$ & $564(100 \%)$ \\
\hline
\end{tabular}




\section{Leishmaniose Visceral Humana (LVH)}

Durante o período de estudo (2009-2012) foram notificados 2.755 casos de suspeita de LVH no estado do Maranhão, desses $1.577(57,24 \%)$ foram confirmados pelo exame de Reação de Imunfluorescência Indireta (RIFI) e 59 (3,74\%) vieram ao óbito. $\mathrm{O}$ ano onde ocorreu um maior número de casos soropositivos foi 2011 , com $465(29,5 \%)$ e 23 (39\%) de óbitos (Figura 3). humana. No ano de 2012 foram notificados 3.038 casos da doença em humanos no Brasil, com uma incidência da ordem de 1,57 casos/100.000 habitantes e uma taxa de letalidade de 7,1\% (MARCONDES e ROSSI, 2013).

Dos casos notificados para a LVH no estado do Maranhão, a maioria era do sexo masculino, em todos os anos do estudo conforme demonstra a figura 4 . Esses dados corroboram com

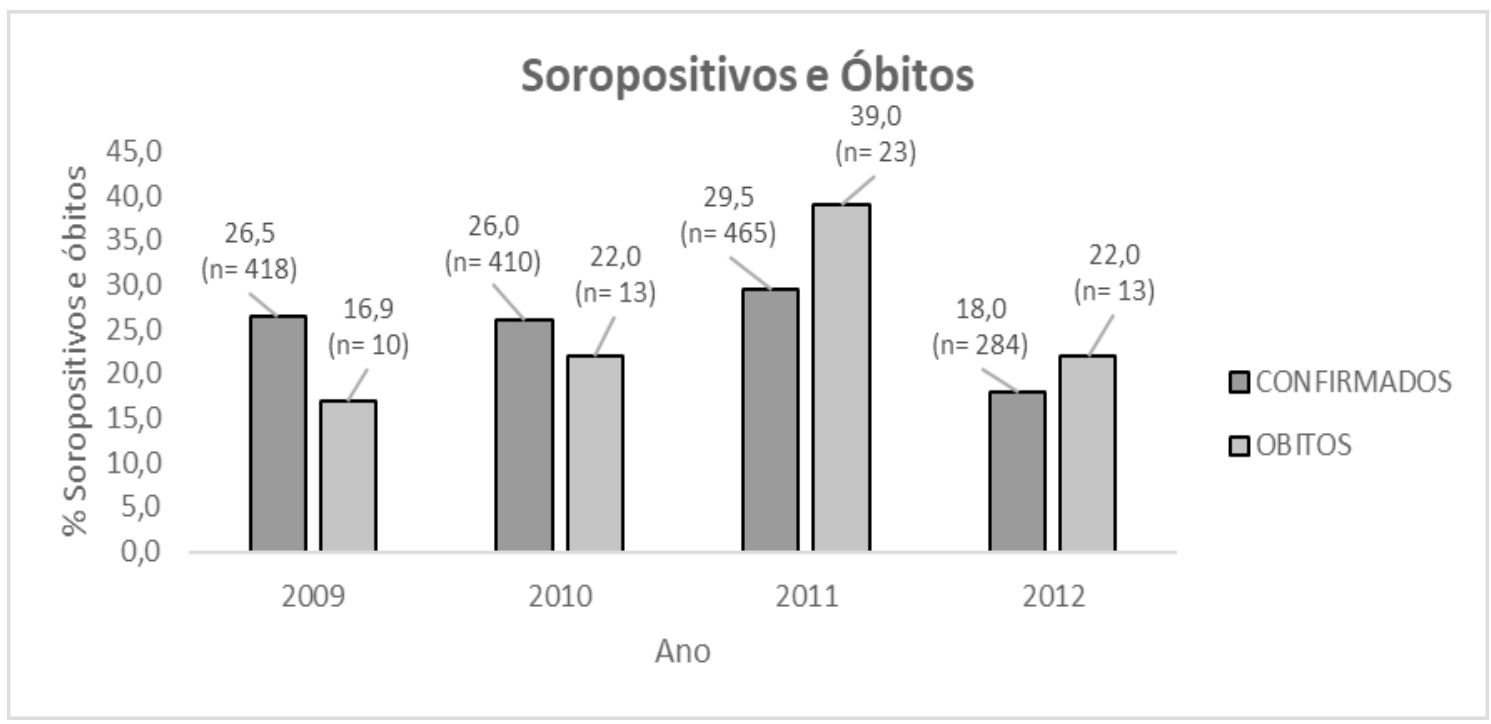

Figura 3: Casos confirmados e óbitos para a LV Humana no estado do Maranhão, no período de 2009 a 2012

No ano de 2009 foram confirmados 418 casos da doença em humanos no Maranhão com uma incidência de 65,65 casos/1.000.000 habitantes; em 2010 a incidência foi de 62,36 casos; em 2011 a incidência foi de 69,97 casos; e no ano de 2012 a incidência foi de 42,30 casos de leishmaniose visceral estudos realizados por Alvarenga et al. (2010), no qual 74,7\% dos pacientes portadores de LVH eram do sexo masculino e $25,3 \%$ do sexo feminino. Apesar da importância epidemiológica e de não estar totalmente esclarecido, a literatura aponta o sexo masculino como mais suscetível à parasitose.

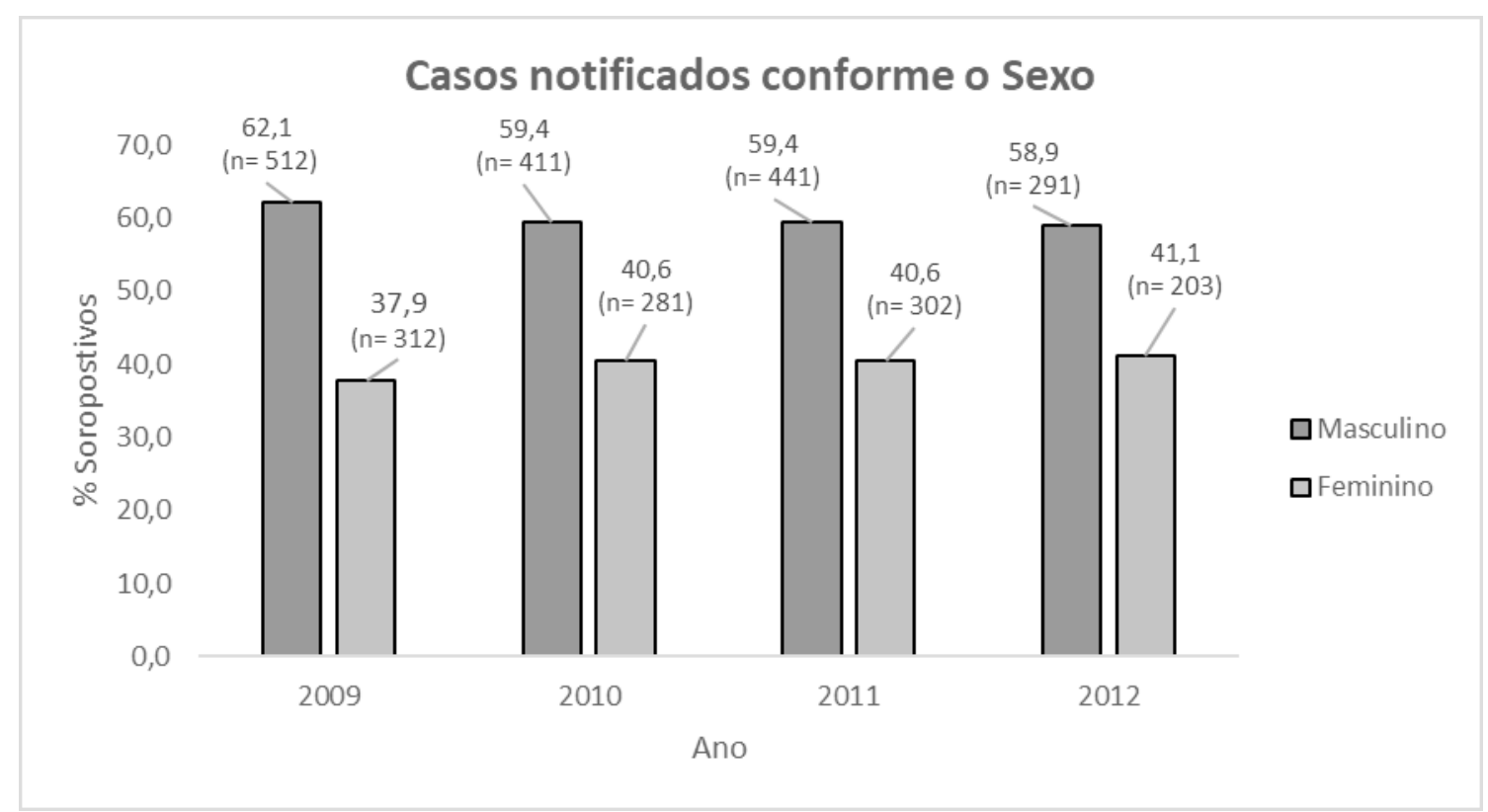

Figura 4: Casos notificados para a LV Humana no estado do Maranhão, segundo o sexo, no período de 2009 a 2012 
A faixa etária onde houve maior ocorrência de casos notificados estava compreendida entre 1-4 anos de idade (Figura 5).
Dessa forma, pode-se observar que a LVH também está presente nos municípios do estado, principalmente em cidades que vêm

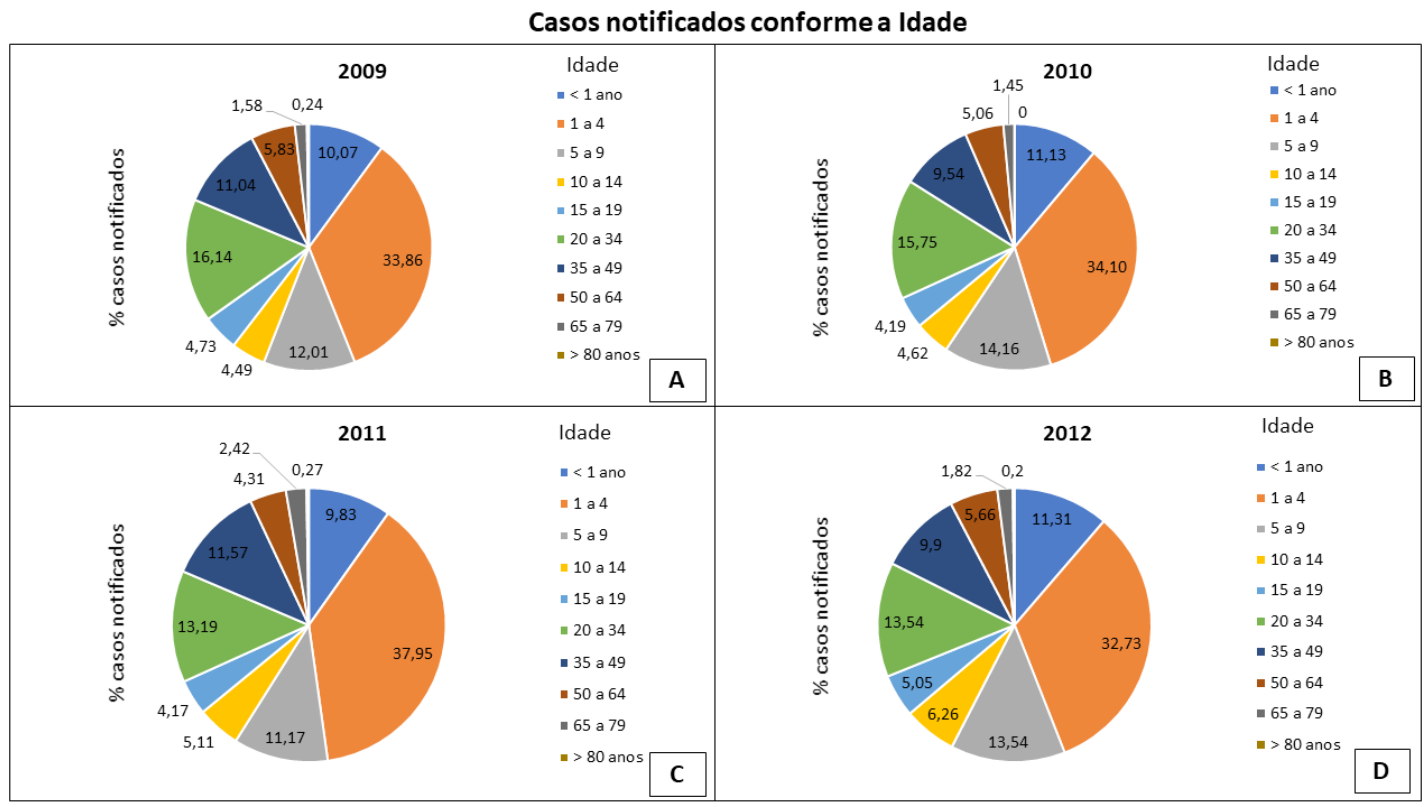

Figura 5: Casos notificados LV Humana no estado do Maranhão, segundo a faixa etária, no período de 2009 a 2012 (A-D).

Estes resultados corroboram com os dados registrados por DantasTorres (2006), de um total de 336 casos registrados no Estado de Pernambuco, $68,5 \%$ eram crianças menores de nove anos.

Verificou-se que a LVH estava presente em 129 municípios do estado do Maranhão no período em estudo, sendo que os de maior frequência foram Imperatriz com 126 (4,57\%) dos casos notificados, em seguida o município de Caxias com 106 (3,85\%), São Luís com 82 (2,98\%), Açailândia com 75 (2,72\%) e o município de Barra do Corda com 60 (2,18\%) dos casos notificados para LVH conforme figura 6. apresentando um elevado índice de crescimento e urbanização, como Imperatriz e Açailândia. Conforme Penha et al. (2013) as mudanças no comportamento humano e no ambiente natural do agente etiológico, do vetor e dos reservatórios exercem grandes impactos no perfil epidemiológico da leishmaniose, tornando-a emergente em algumas áreas urbanas.

Nestes municípios, embora a urbanização e desenvolvimento, o saneamento básico ainda é muito precário ou inexistente com criadouros ou acúmulos de lixo em vários bairros, presença de abrigos de animais silvestres dentro do perímetro, a

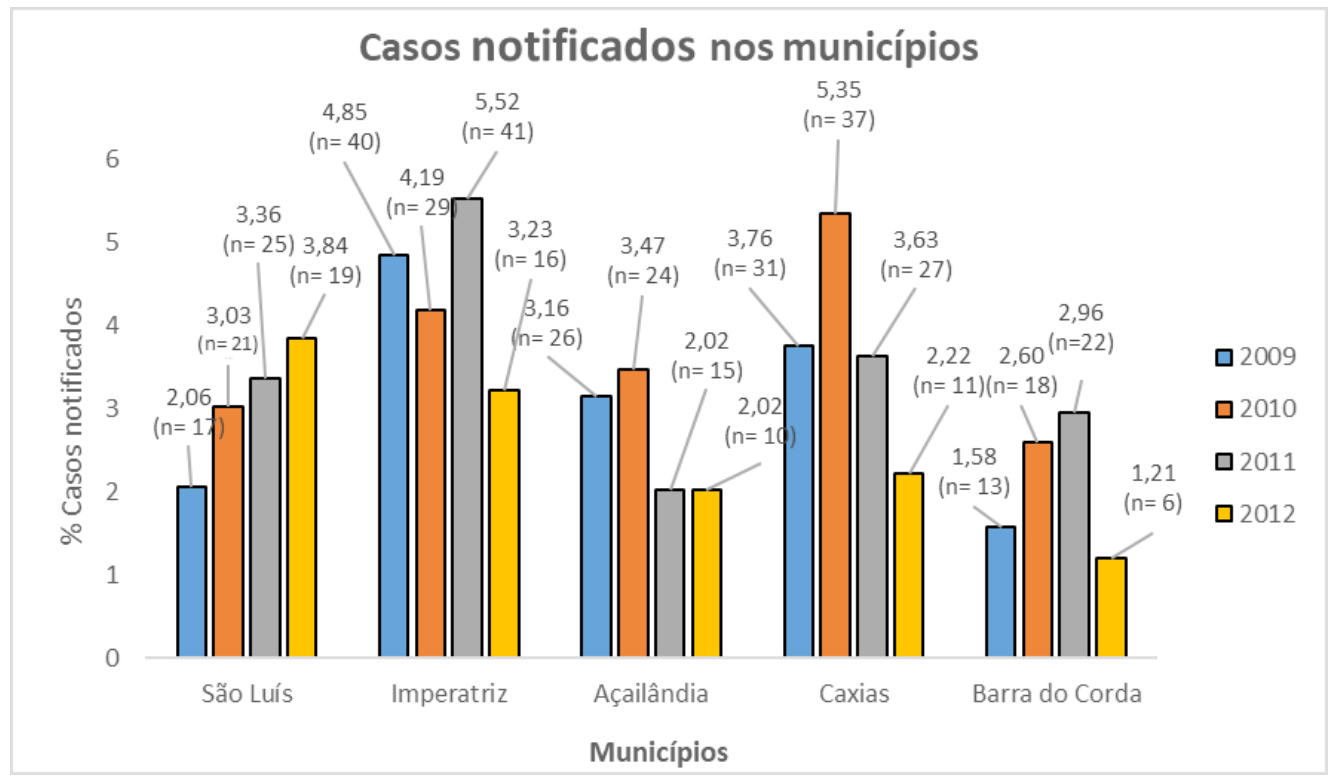

Figura 6: Casos notificados LV Humana nos municípios do estado do Maranhão, no período de 2009 a 2012 
arborização abundante em quintais, descontinuidade do serviço de entomologia e diminuição das operações com inseticida pelos órgãos competentes (COSTA et al., 1995). Na figura abaixo, apresenta a prevalência da LVH nas microrregiões do estado do Maranhão. maior frequência 398 (53,57\%) (Tabela 2). Este dado confirma o que diz Monteiro et al. (2005), "que a LV vem ocorrendo com mais frequência em áreas quentes, onde o clima é seco com média de chuvas anuais de $550 \mathrm{~mm}^{2}$ ", aspectos semelhantes ao encontrado no estado em estudo.
2009

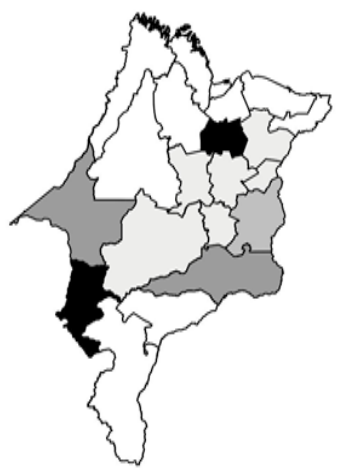

$100 \quad 0100 \mathrm{~km}$

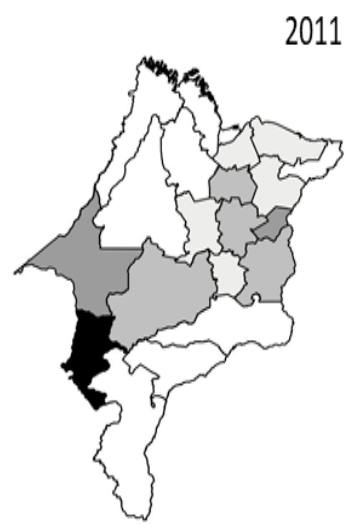

$100 \quad 0 \quad 100 \mathrm{~km}$
Legenda

Prevalência

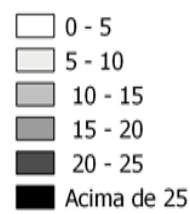

Legenda

Prevalência

$\square$ - 5

$\square$ - -10

$\square 10-15$

$\square 15-20$

$\square 20-25$

Acima de 25

Figura 7: Prevalência da LV Humana nas microrregiões do estado do Maranhão, no período de 2009 a 2012

O estado do Maranhão apresenta 21 microrregiões, destas a LVH esteve presente em vinte, e somente na microrregião Gerais de Balsas que não houve notificação de caso no período em estudo. Nesta figura observa-se que a maior prevalência da LVH localizou-se na microrregião de Porto Franco no centro-sul do estado e em Itapecuru Mirim no norte maranhense com registro acima de 25 casos por 100.000 mil habitantes no período em estudo. No ano de 2012, houve uma diminuição da prevalência, sendo registrada em menor número de casos nas microrregiões localizadas no centra-oeste do estado.

O período chuvoso foi onde ocorreu uma maior frequência de notificações de suspeita da LVH, sendo o ano de $2011 \mathrm{com}$
Tabela 2: Casos notificados de LV Humana no estado do Maranhão, segundo época do ano, no período de 2009 a 2012

\begin{tabular}{lccc}
\hline Anos & $\begin{array}{c}\text { Período chuvoso } \\
\text { N (\%) }\end{array}$ & $\begin{array}{c}\text { Período seco } \\
\text { N (\%) }\end{array}$ & $\begin{array}{c}\text { Total } \\
\text { N (\%) }\end{array}$ \\
\hline 2009 & $411(48,81 \%)$ & $413(51,19 \%)$ & $824(100 \%)$ \\
2010 & $348(50,29 \%)$ & $344(49,71 \%)$ & $692(100 \%)$ \\
2011 & $398(53,57 \%)$ & $345(46,43 \%)$ & $743(100 \%)$ \\
2012 & $260(52,42 \%)$ & $235(47,47 \%)$ & $495(100 \%)$ \\
Total & $\mathbf{1 . 4 1 7 ( 5 1 , 4 5 \% )}$ & $\mathbf{1 . 3 3 7 ( 4 8 , 5 5 \% )}$ & $\mathbf{2 . 7 5 4 ( 1 0 0 \% )}$ \\
\hline
\end{tabular}




\section{Conclusão}

Conclui-se que o estado do Maranhão apresenta uma área endêmica para a Leishmaniose Visceral, com registros de caso desde a década de 80 até o presente estudo. Neste estudo o sexo masculino foi o mais afetado, assim como os machos nos caninos. Os municípios mais atingidos pela LV humana foram os mais desenvolvidos e com maior urbanização, embora tenham um melhor acesso ao sistema de saúde em relação aos municípios rurais e menos desenvolvidos, a ausência de saneamento básico a destruição de ecótopos silvestres favorece a procura do vetor por outras fontes alimentares humanas e animais.

\section{Referências}

ALVARENGA, D.G; ESCALDA, P.M.F.; COSTA, A.S.V.; MONREAL, M.T.F.D. Leishmaniose visceral: estudo retrospectivo de fatores associados à letalidade. Revista da Sociedade Brasileira de Medicina Tropical, v. 43, n. 2, p. 194-197, mar-abr, 2010.

AMORA, S.S.A,; SANTOS, M.J.P.; ALVES, N.D.; COSTA, S.C.G.; CALABRESE, K.S.; MONTEIRO, A.J.; ROCHA, M.F.G. Fatores relacionados com a positividade de cães para leishmaniose visceral em área endêmica do Estado do Rio Grande do Norte, Brasil. Ciência Rural, v.36, n.6, p.1854-1859, nov-dez, 2006.

BARBOSA, D.S.; ROCHA, A.L.; SANTANA, A.A.; SOUZA, C.S.F.; DIAS, R.A.; COSTA-JÚNIOR, L.M.; ABREU-SILVA, A.L. A. Soroprevalência e variáveis epidemiológicas associadas à leishmaniose visceral canina em área endêmica no município de São Luís, Maranhão, Brasil. Ciência Animal Brasileira, v. 11, n. 3, p. 653-659, jul-set, 2010.

Brasil. Manual de vigilância e controle da leishmaniose visceral. Ministério da Saúde, Brasília, 2006.

CAMARGO, T.C.; BONDAN, E.F. Conhecimento sobre leishmaniose visceral canina na população do município de Cotia (SP), Brasil, e participação dos clínicos veterinários locais na propagação de medidas preventivas. Revista brasileira Ciência Veterinária, v. 22, n. 1, p. 28-33, jan./mar., 2015.

CIARAMELLA, P.; CORONA, M. Canine leishmaniasis: therapeutic aspects. Compendium, v. 25, p. 370-375, 2003.

COSTA, C.H.N. How effective is dog culling in controlling zoonotic visceral leishamniasis? A critical evaluation of the science, politics and ethics behind this public health policy. Revista da Sociedade Brasileira de Medicina Tropical, v.44, n.2, p.232-242, 2011.

COSTA, J.M.L; VIANA, G.M.C.; SALDANHA, A.C.R.;

NASCIMENTO, M.D.S.B.; ALVIM, A. C.; BURATTINI, M.N.; SILVA, A.R. Leishmaniose Visceral no Estado do Maranhão, Brasil. A Evolução de Uma Epidemia. Caderno de Saúde Pública, Rio de Janeiro, v. 11, n. 2, p. 321-324, Abr/Jun, 1995.

DANTAS-TORRES, F. Situação atual da epidemiologia da leishmaniose visceral em Pernambuco. Revista Saúde Pública, v. 40 , n. 3, p. 537-41.

FARIA, A.R.; ANDRADE, H.M. Diagnóstico da leishmaniose visceral canina: grandes avanços tecnológicos e baixa aplicação prática. Revista Pan-Amazônica Saúde, v.3, n.2, p.47-57, 2012.

GUIMARÃES, K.S.; BATISTA, Z.S.; DIAS, E.L.; GUERRA; R.M.N.C.; COSTA, A.D.C.C.; OLIVEIRA, A.S.; CALABRESE; K.S.; CARDOSO; F.O.; SOUZA, C.S.F.; ZAVERUCHA DO VALE T.; GONÇALVES DA COSTA, S.C.; ABREU-SILVA, A.L. Canine visceral leishmaniasis in São José de Ribamar, Maranhão State, Brazil. Veterinary Parasitology, v. 131, p. 305-309, 2005.
O cão é considerado o principal reservatório urbano da doença, pois este alberga o agente e apresenta grande significância no ambiente familiar, dessa forma o combate ao vetor e eutanásia de cães soropositivos são as principais medidas para combater a doença no Estado. Além disso, detecção de casos suspeitos, não viajar com os cães para áreas endêmicas, implantação de programas de controle de natalidade para cães, aprimoramento dos métodos de detecção da LVC, tratamento dos animais infectados e campanhas públicas são ações importantes de combate da Leishmaniose.

IKEDA-GARCIA, F.A.; MARCONDES, M. Métodos de diagnóstico da leishmaniose visceral canina. Clínica Veterinária, São Paulo, ano 12, n. 71, p.34-42, 2007.

MARCONDES, M.; ROSSI, C.N. Leishmaniose visceral no Brasil. Brazilian Journal Veterinary Research. Animal Science, São Paulo, v. 50, n. 5, p. 341-352, 2013.

MATOS, M.M.; FILGUEIRA, K.D.; AMORA, S.S.A.; SUASSUNA, A.C.D.; AHID, S.M.M.; ALVES, N.D. Ocorrência da leishmaniose visceral em cães em Mossoró, Rio Grande do Norte. Ciência Animal, v. 16, n. 1, p. 51-54, 2006.

MENDES, S.W.; SILVA, A.A.M.; TROVÂO, J.R.; SILVA, A.R.; COSTA J.M.L. Expansão espacial da Leishmaniose visceral Americana em São Luís do Maranhão, Brasil. Revista da Sociedade Brasileira de Medicina Tropical, v. 35, p. 227-231, 2002.

MONTEIRO, E.M.; SILVA, J.C.R.; COSTA, R.T.; COSTA, D.C.; BARATA, R.A.; PAULA, E.V.; MACHADO-COELHO, G.L.L.; ROCHA, M. F.; FORTES-DIAS, C.L.; DIAS, E.S. Leishmaniose visceral: estudo de flebotomíneos e infecção canina em Montes Claros, Minas Gerais. Revista da Sociedade Brasileira de Medicina Tropical, Uberaba, v.38, n.2, p.147-152, 2005.

NASCIMENTO-ROCHA, J.M; SANTOS, H.D.; ABOUD-DUTRA, A.E.; GAZÊTA, G.S.; BARBOSA, S.M.; FLORENTINO, C.M. Leishmaniose Visceral em cão domiciliado de Araguaína I Tocantins. Revista brasileira Ciência Veterinária, v. 9, n. 2, p. 120122, maio/ago. 2002.

NEVES, D. P. et. al. Parasitologia Humana. São Paulo, Editora Atheneu, 1997, $524 \mathrm{p}$.

PENHA, T.A.; SANTOS, A.C.G.; REBÊLO, J.M.M.; MORAES, J.L.P; GUERRA, R.M.S.N.C. Fauna de flebotomíneos (Diptera: Psychodidae) em área endêmica de leishmaniose visceral canina na região metropolitana de São Luís - MA, Brasil. Biotemas, v. 26, n. 2, p. 121-127, junho, 2013.

SCHIMMING, B.C.; SILVA, J.R.C.P. Leishmaniose visceral canina - Revisão de literatura. Revista científica eletrônica de medicina veterinária. n.19, ano X, julho, 2012.

VERONESI, R.; FOCACCIA, R. Tratado de Infectologia. São Paulo: Editora Roca Ltda, 2003, 353 p. 